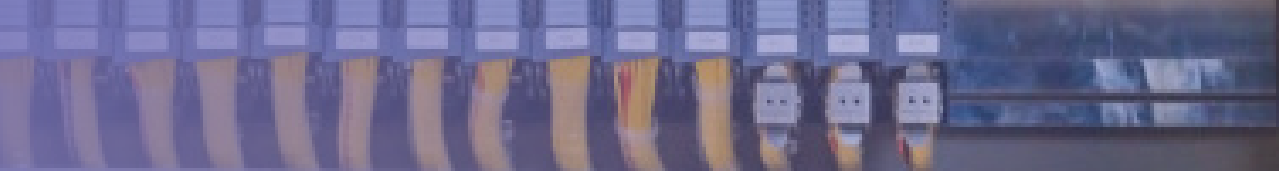

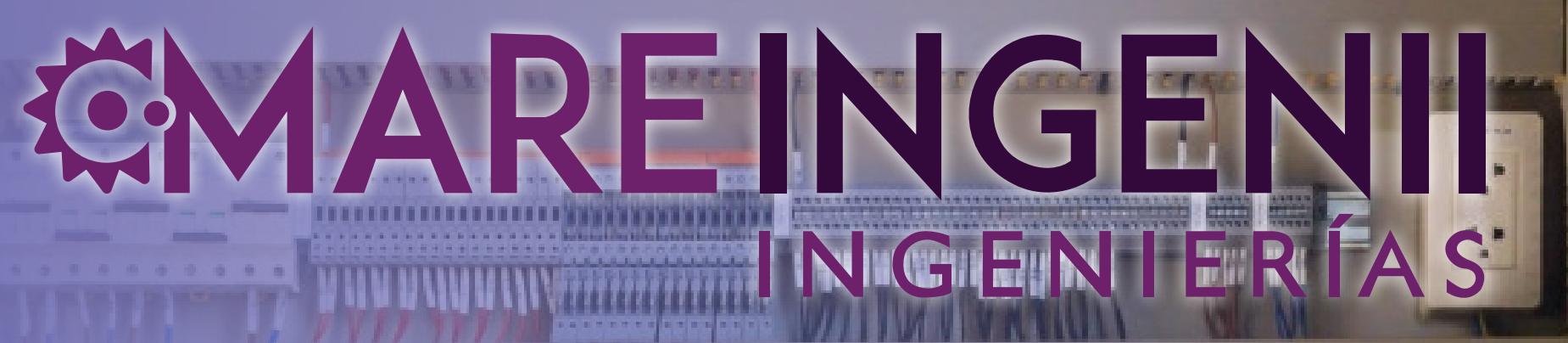

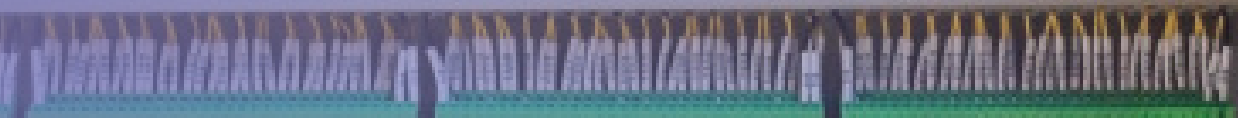

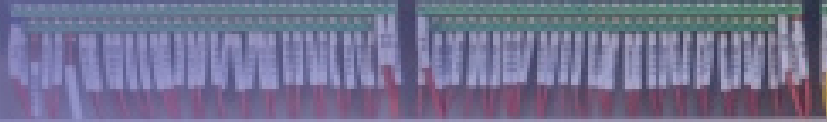

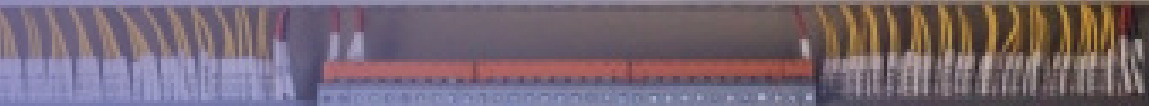

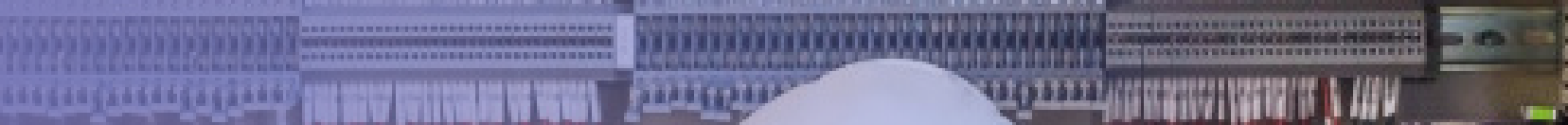

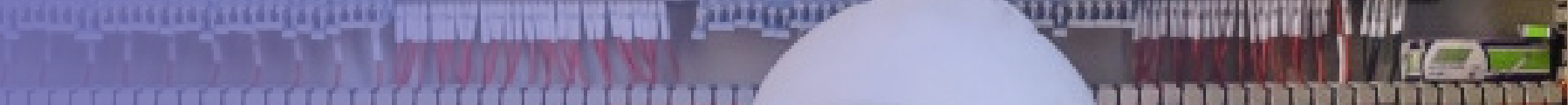

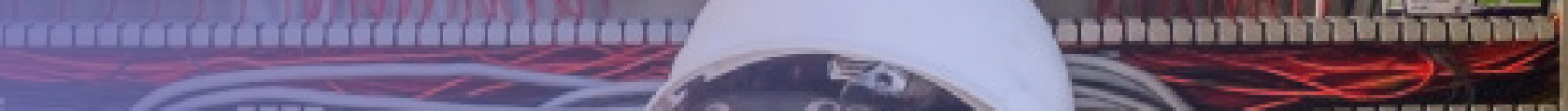

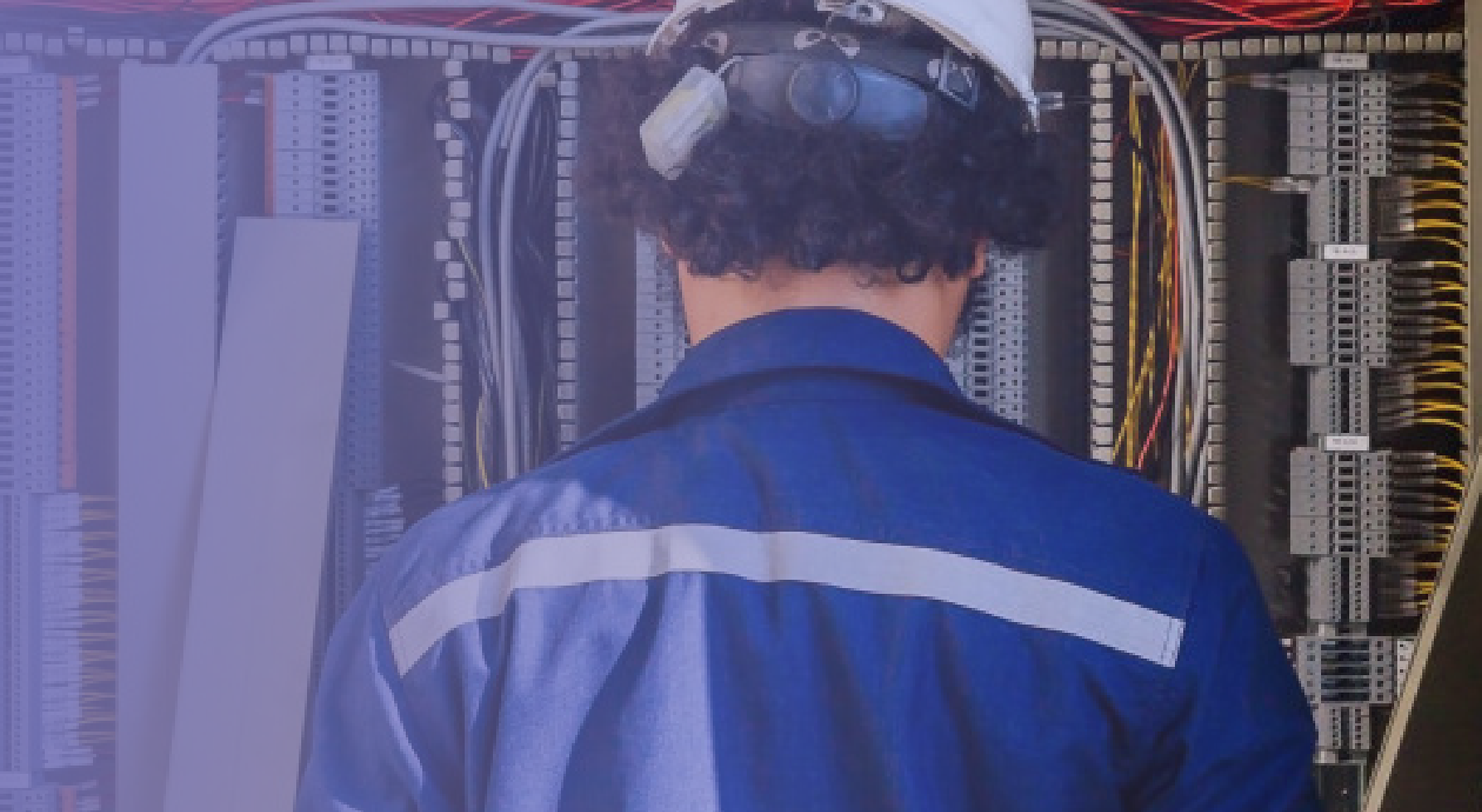

Ingeniería en Telecomunicaciones 


\title{
Tecnología móvil 5G
}

\author{
5G mobile technology
}

Moisés Elías Batista Fuentes'; Eliana Katherine Díaz Ibáñez²

${ }^{1}$ Fundación Universitaria San Mateo. Correo electrónico: mebautista@funsanmateo.edu.co

${ }^{2}$ Fundación Universitaria San Mateo. Correo electrónico: ekdiaz@funsanmateo.edu.co

Como citar: Batista Fuentes, M; Díaz Ibáñez, E. 2019. Tecnología móvil 5G. Mare Ingenii. Ingenierías 1(1). Disponible en http://cipres.sanmateo.edu.co/index.php/mi

Recibido: Junio 19 de 2019

Aceptado: Septiembre 11 de 2019

\section{RESUMEN}

A través de las generaciones, la tecnología ha permitido avanzar y brindar sus maravillosos beneficios. Para la vida cotidiana abre una brecha para ofrecernos aún más beneficios. El objetivo de este artículo es describir una propuesta para la tecnología móvil, a saber, la llamada 5G que permitirá mostrar al mundo avances como la realidad aumentada (RA), la realidad virtual (RV), las ciudades inteligentes y la gran innovación del Internet of Things (IOT).

Palabras clave: internet de las cosas; espectro electromagnético; radioeléctrico; onda milimétrica; $5 G$.
ABSTRACT

Trough generations, the technology has allowed advance and give to the world its greats benefits. For our daily life, it opens a gap to offer even more benefits to the society. The objective of the present article is to describe a purpose for mobile technology, namely the 5G. That will allow us show to the world advances as augmented reality $(A R)$, virtual reality $(R V)$, smart cities and the big innovation: the Internet of Things (IoT).

Keywords: internet of things; electromagnetic spectrum; radioelectric spectrum; millimeter band; $5 G$. 


\section{INTRODUCCIÓN}

Cada nueva generación de redes móviles o inalámbricas trae consigo mayor velocidad y mejores funcionalidades. La tecnología de la conexión inalámbrica comenzó brindando servicio de solo voz denominada 1G; luego pasó a la 2G brindando servicios de voz y mensajería instantánea (SMS). Con la evolución, más adelante, pasa a la tecnología 3G permitiendo realizar llamadas, enviar mensajes y navegar por internet (conexión en línea). Con el tiempo 3G evoluciona a 4G brindando los mismos servicios, pero a mejor velocidad. Más tarde con la evolución de la 4G pasa a 4G LTE, una versión más consistente y más rápida que la misma 4G. [1].

La evolución continúa y se introducen mejoras en las antenas, en su capacidad, cobertura, espectro y calidad de señal surgiendo la nueva tecnología 5G mostrando sus características y prestando una mejora en la velocidad de transferencia de datos. Así, la velocidad de conexión a Internet empieza a ser mucho mejor y más eficaz, prometiendo mejorar la capacidad de respuesta de las redes inalámbricas. Es de considerar que hoy en día el Internet of Things(IoT) ha tenido ungran crecimiento y auge en la conexión a innumerables dispositivos inteligentes y servicios automatizados. De una red con prestaciones de bastante capacidad, cobertura y velocidad, es preciso que soporte y satisfaga los requerimientos y necesidades de las conexiones y el usuario final. Para ello es la nueva generación de la tecnología móvil 5G.

En realidad, para tener una red $5 \mathrm{G}$ con una buena conectividad se debe implementar una buena infraestructura o mejorar la existente y contar con un buen espectro de transmisión. Cabe aclarar que la tecnología 5G aún está en desarrollo y en fase de pruebas.

En este artículo divulgativo, se pretende describir las características de la red 5G y las posibles ventajas de las prestaciones que trae consigo esta nueva generación de conexión móvil. Lo anterior, a partir del punto de vista de tres ingenieros expertos en el tema Mahesh Choudhary, William Sánchez y Aldo Schenone, ponentes de la convención internacional en ingeniería electrónica, eléctrica y de sistemas (INTERCON) en Lima, Perú, desde el área de las telecomunicaciones. A través de sus postulados, se evidencian las características y requerimientos del $5 \mathrm{G}$.

\section{¿QUÉ ES 5G?}

$5 G$ es la quinta generación de redes de comunicaciones móviles basada y cimentada en la red 4 GLTE. Permite realizar varios servicios de forma más rápida y eficaz con una velocidad de transferencia de 5000 megabits por segundo aproximadamente, mucho mayor que la 4GLTE. Además cuenta con una gran estabilidad de conexión e interacciones con el loT. [2].

Debido a que entre más usuarios se conectan a la red, la tecnología 4G ha venido alcanzando su límite de capacidad. Frente a esto, no es un secreto que los usuarios entre mejor servicio tienen, quieren más velocidad y menos tiempo de espera. Por eso llega la 5G, la nueva generación de redes inalámbricas, capaz de soportar 1000 veces más el tráfico que la red actual de 4G y será diez (10) veces más rápido que la red $4 \mathrm{G}$ LTE. $5 \mathrm{G}$ será la base esencial para proyectos de realidad virtual, conducción autónoma, IOT, automatización de sistemas y mucho más.

A partir de varios ponentes sobre el tema $5 \mathrm{G}$ en la convención de la INTERCON realizada en Lima, Perú, en el año 2018, el ingeniero en soluciones de red inalámbrica en América Latina de la empresa Huawei, Mahesh Choudhary, expresa que:

[...] una de las mejoras con la tecnología $5 G$ es que tendrá mayor capacidad de transmisión. Alta definición en los videos en $4 k$ y $8 k$ ya que requieren más velocidad. Fiabilidad en el acceso y conexión con todos los dispositivos móviles mejorando la experiencia en los servicios. Aumento en la velocidad de las aplicaciones y transferencia de datos, incrementando la posibilidad de más prestación de servicios. Baja latencia en la comunicación y transferencia de datos. Con la nueva tecnología $5 G$ se abrirá nuevas oportunidades de negocios y proyectos en marcha como robótica inalámbrica, navegación de drones, exploración de realidad virtual, etc. [3].

A partir del punto de vista de Mahesh Choudhary, para la llegada de la generación 5G se necesita implementar o contar con una multibanda, una multicanal y dispositivos de Tecnología de Acceso de radio Multiple (multi-RAT, Multiple Radio Access Technology), dispositivos móviles que se pueden conectar a más de un tipo de red celular.Lo anterior, para satisfacer y soportar el tráfico, los usuarios y los servicios de esta nueva tecnología que vendrá de la mano con el loT. 
En países como Corea y Puerto Rico, así como en ciudades tales como Vancouver ya están implementando estas consideraciones para esta tecnología. La red de telecomunicaciones 5 G llegará al mercado hacia el año 2020 aproximadamente.

\section{VISIÓN TÉCNICA DEL 5G}

Por otra parte, el gerente de cuenta de la empresa Nokia, el ingeniero William Sánchez, menciona y complementa que "la tecnología $5 \mathrm{G}$ revolucionará el ámbito de las telecomunicaciones, debido a gran velocidad de transferencia que se manejará" [4] Esta tecnología permitirá una trasferencia de datos máximos de 10 Gigabit por segundo (Gbps), con más capacidad, cobertura, menos espacio y ahorro de energía, mejorando los procesos industriales, administrativos y de negocios. Sánchez argumenta que se debe tener en cuenta cinco componentes claves en la implementación de la tecnología 5G y sus productos, para lograr el objetivo esperado. Estos componentes son:

- Nuevas opciones de espectro: es un punto importante, allí se asignan las bandas de frecuencia radioeléctrica de transmisión. Este debe ser de bastante capacidad para un correcto funcionamiento y buen servicio, contando con mayor velocidad de conexión, menor latencia y una mejor eficiencia en su desempeño. Como nota de referencia, el espectro radioeléctrico es el segmento que comprende el rango de los $3 \mathrm{KHz}$ y los $300 \mathrm{GHz}$ utilizada para la transmisión de tecnologías inalámbricas.

- Masivas MIMO (múltiples entradas múltiples salidas): para una mayor cobertura en zonas de difícil acceso y evitar pérdidas de paquetes. Mediante la utilización de varias antenas de manera simultánea, proporciona un aumento en la velocidad de transmisión. [5].

- Nuevo diseño de radio y marco flexible: se refiere al control de tamaño del radio y marco flexible de la onda mediante la multiplexación por división de frecuencia ortogonal (OFDM), un método muy utilizado para la transmisión inalámbrica de alta velocidad de datos. Este, combinado con arreglos de antenas tanto en el emisor como en el receptor, aumenta la ganancia y mejora la capacidad del sistema en canales variables y selectivos en frecuencia (MIMO). Asimismo se requiere de un ancho de banda mayor que el de 4G. 5G podrá alcanzar los $20 \mathrm{~Gb} / \mathrm{s}$ aproximadamente, permitiendo el desarrollo del vídeo en línea, realidad virtual, hologramas, etc. El ingeniero Sánchez (2018) indica que "el reto del 5G es la explotación del loT en campos tan variados como sensores, sanidad, transporte, máquinas e industrialización" [4].

- Agregación y Conectividad múltiple: 5G permite aprovechar el mundo del IoT. Busca unir las diferentes tecnologías que utilizan los operadores permitiendo el intercambio de información entre ellas logrando múltiples conexiones.

- Distribución flexible de la arquitectura: con el 5G se pretende una virtualización de la Red de Acceso por Radio (RAN), logrando mejorar y fortalecer el Single RAN "en la que misma estación base puede operar con distintas tecnologías y arquitecturas: 2G, 3G, 4G, LTE y en el futuro 5G" [4].

\section{NON-STANDALONE (NSA) Y STANDALONE (SA)}

Por un lado, Non-standalone (NSA) es una interfaz de aire no autónoma que permite conectar redes 2G, 3G, 4G y 5G simultáneamente en base del estándar 3gpp. Alcanza velocidades superiores a dos (2) Gbps con latencias inferiores a diez (10) ms. Esta tecnología será la utilizada para los despliegues comerciales del 5G. Por otro lado, Standalone (SA), es una interfaz de aire autónoma que solo permite la conexión de redes $5 \mathrm{G}$ de forma independiente, es decir, no se conecta a antenas 2G, 3G o 4G. Para la nueva generación de conexiones móviles $5 \mathrm{G}$ se ha venido desarrollando pruebas con estándares NSA 5g, New Radio (NR). La entidad 3Gpp ha aprobado especificaciones técnicas para este estándar que se apoyará en las redes existentes de $4 \mathrm{G}$ LTE. Esto significa que la red $5 G$ será compatible con la infraestructura $4 G$, ofreciendo portadoras de $5 G$ en la interfaz de aire, brindando mayor velocidad de transferencia y menor latencia.

Asimismo el estándar SA aún está en validación y en pruebas. Dicho estándar requiere una infraestructura nueva que solo permite el uso de redes 5G, es decir, este estándar no se conecta 
a antenas 2G, 3G, 4G. Una vez aprobado este estándar por la 3Gpp, la transición de 5G NSA a 5G SA será invisible y no tendrá problema por parte de los operadores [6].

\section{CASOS DE USO DE LA 5G}

Mediante el punto de vista del ingeniero William Sánchez, indica tres pilares de la visión de conjunto del Servicio de la tecnología 5G:

- Banda Ancha Móvil Mejorada (eMBB): facilitará velocidades en movilidad superiores a $100 \mathrm{Mbit} / \mathrm{s}$ con picos de un (1) Gbit/s. Permitirá, por ejemplo, ofrecer contenidos en ultra alta definición - experiencias de realidad virtual. También, posibilitará casos de uso impulsados por datos que requieren altas velocidades de datos en un área de cobertura amplia lo que garantizaría una mayor capacidad, conectividad mejorada y mayor movilidad del usuario [7].

- Comunicaciones ultra confiables de baja latencia (URLLC): requisitos estrictos de latencia a un (1) milisegundo (ms) frente a 20-30 ms propios de las redes 4G.

Esta condición podría hacerlas apropiadas para aplicaciones de comunicaciones de misión crítica que tienen requerimientos específicos en este ámbito, como el vehículo conectado o el vehículo autónomo, servicios de telemedicina, sistemas de seguridad y control en tiempo real, cirugía remota, internet táctil y otros como la fabricación inteligente [7].

- Comunicaciones masivas tipo máquina a máquina (mMTC): se incrementará la capacidad para gestionar gran cantidad de conexiones simultáneas. Permitirá entre otras cosas, el despliegue masivo de sensores, el loT y el crecimiento de los servicios de big data [7].

Para cumplir los requisitos o servicios anteriores, se espera que $5 \mathrm{G}$ respalde:

- La capacidad de tráfico de 10Mbps por metro cuadrado en áreas con puntos críticos.
- Las tasas de transferencia de datos experimentadas por el usuario de hasta un (1) Gbps, con tasas de transferencia de datos máximas en decenas de Gbps y un volumen de tráfico completo de al menos un (1) Tbps por kilómetro cuadrado.

- La latencia de un (1) ms para el intercambio de datos experimentado por el usuario.

- Densidad de conexión de hasta un millón de conexiones por kilómetro cuadrado.

- Una alta movilidad de hasta $500 \mathrm{~km} / \mathrm{hora}$ en trenes de alta velocidad y hasta $1.000 \mathrm{~km} / \mathrm{hora}$ en aviones, con una experiencia de usuario mejorada [7].

Unas de las incógnitas que surge del tema es ¿por qué 5G?, el ingeniero de telecomunicaciones William Sánchez de la empresa Nokia, responde brevemente a esta incógnita indicando:

"Debido a los nuevos servicios, conexiones y casos de uso significativos de IoT por la cual requieren más velocidad y menos latencia, mejorando los servicios inalámbricos fijos. Esta nueva generación de comunicaciones móviles puede iniciarse con la tecnología madura existente especialmente la 4G brindando las prestaciones requeridas, 5G se volverá obligatorio en todo el ámbito de conexión y acceso a servicios loT y mientras que la necesidad de capacidad aumenta la latencia disminuye" [4].

Por otra parte, Nokia y Qualcomm completaron la primera llamada utilizando 3gpp, compatible con la nueva tecnología de radio 5G. Esta prueba fue exitosa en el espectro de ondas milimétricas de Verizon utilizando la tecnología de red Nokia $5 G$, en un dispositivo prototipo $5 G$ NR provisto por Qualcomm Technologies. Esta prueba se realizó en las instalaciones de Nokia en Murray Hill, Nueva Jersey. Aún hoy siguen las pruebas de interoperabilidad previas entre Nokia y Qualcomm.

En Corea, en el mundial del 2017, se realizaron pruebas con las soluciones de tecnología 5G. Allí, las personas afuera del estadio se colocaron unas gafas de realidad virtual que hacía vivir la experiencia como si estuvieran dentro del estadio en la tribuna.

Esto da a conocer las dimensiones que puede prestar 5G. El ingeniero Sánchez argumenta que "el tema de la $5 \mathrm{G}$ se requiere de mucha planeación, infraestructura, tiempo y precisión para que llegue con las prestaciones y expectativas requeridas." 


\section{ONDA MILIMÉTRICA}

Las prestaciones y servicios de la próxima generación requerirán una capacidad inmensa, enormes tasas de transmisión de información y el incremento del tráfico. Lo previamente mencionado conlleva a requerir velocidades de transferencia diez veces más que la habitual, es decir, diez (10) gigabits por segundo e incrementos del ancho de banda que las que ofrece la tecnología 4G.

Para lograr suplir las expectativas de la nueva generación 5G, se empleará ondas milimétricas (mmwave) presentes en las implementaciones multi-RAT. En el proyecto red de acceso de radio móvil basado en ondas milimétricas para comunicaciones integradas de quinta generación (mmMAGIC), los principales proveedores de infraestructuras y equipamientos, centros de investigación y universidades junto con algunas pymes de Europa, se asociaron para resolver esas dudas.

Convencidos de que las redes móviles que operan en bandas de onda milimétrica deberían ser capaces de operar como redes independientes (single-RAT), las implementaciones multi-RAT desarrollaron una interfaz de radio de onda milimétrica para abordar los retos específicos de la comunicación de onda milimétrica y conseguir la capacidad, el rendimiento, la latencia y la eficiencia energética necesarios para prestar servicios 5G. mmMAGIC estuvo a la vanguardia de la investigación y el desarrollo de nuevas tecnologías de acceso por radio (RAT), en las bandas de frecuencia de onda milimétrica (mm-wave) (6-100 $\mathrm{GHz}$ [8].

\section{TECNOLOGÍAS QUE EMERGIERON DEL}

\section{G}

Es mucho el avance de la red 5G. A partir de este, han surgido avances tecnológicos que facilitan aún más las conexiones inalámbricas de esta generación tales como:

- Ondas Milimétricas.

- Celdas pequeñas.

- Massive MIMO.

- Beamforming (sistema de señalización de tráfico).

- Full Duplex.

\section{VENTAJAS Y DESVENTAJAS DEL 5G}

Ventajas

- Aumento en el rendimiento de transferencia.

- Velocidad de transferencia de datos en downlink de diez (10) Gbps.

- Baja latencia de uno (1) a cinco (5) milisegundo en la transmisión.

- Tecnología ideal y compatible con loT conectando todo tipo de sensores y dispositivos inteligentes.

- Menor consumo de energía hasta un 90\%.

- Innovación y automatización de la industria.

- Más dispositivos conectados al mismo tiempo.

- Garantizara el 100\% de cobertura.

- Contará con un ancho de banda de 1000 por unidad de área.

- Vida útil de 10 años para dispositivos IoT.

- Viaja en ondas de muy alta frecuencia.

\section{Desventajas}

- Baja capacidad de atravesar obstáculos requiriendo transmisores para rodearlos.

- Algunos dispositivos no serán compatibles con la red 5G. Se requiere el cambio de estos a la compatibilidad con la nueva tecnología.

- El costo de la infraestructura para desplegar el $5 \mathrm{G}$ es elevado.

- Las bandas del espectro para la red serán costosas.

\section{ESTÁNDAR IEEE DE LA 5G}

La nueva tecnología 5G esta implementada en la norma IEEE 802.11ac Wave 2. Esta norma es una mejora de la $802.11 \mathrm{n}$, mejorando el rendimiento a un máximo de 3.47 Gbps [9]. Asimismo 802.11ac Wave 2 incorpora una mejora de la tecnología MIMO llamada Multi-Usuario Múltiple Entrada Múltiple Salida (MU-MIMO) que tiene la capacidad de aumentar la eficiencia de las soluciones wifi.

MU-MIMO utiliza la tecnología beamforming (un método de manejar la señal de radiofrecuencia a través de un access point) para focalizar la señal en los clientes inalámbricos. Además, es capaz de permitir transmisiones simultáneas a los clientes. 
Con la tecnología MU-MIMO, un router permite enviar 1-stream de datos a cada uno de los clientes, por lo que se enviarán los datos simultáneamente y el ancho de banda se aprovechará al máximo. Los tres clientes enviarán y recibirán datos a la vez (en paralelo) y no en serie como ocurre con la tecnología SU-MIMO (Single-User MIMO).

La tecnología MU-MIMO es ideal para el IoT, así como para servicios en tiempo real como videoconferencias y juegos online ya que se transmitirán rápidamente. 5G contará en su bloque de seguridad con un Modulo de Seguridad de Hardware (MSH). Este es un dispositivo criptográfico basado en hardware que genera, procesa, almacena y protege claves criptográficas dentro de dispositivos fortalecidos y resistentes a la manipulación de forma segura [10], [11].

\section{OBJETIVO DE LA RED 5G}

Esta generación de comunicaciones móviles, aparte de mejorar y brindar mayores servicios, busca conseguir más velocidad en la transmisión de datos. También posibilita un ecosistema capaz de entregar eficientemente servicios inalámbricos entre máquinas, cosas y personas revolucionando en todo sentido la forma de comunicarnos mediante conexiones fiables, eficientes y eficaces.

\section{CONCLUSIONES}

Desde la década de los setenta, la tecnología móvil está apenas ofreciendo servicio de voz conocida como tecnología analógica 1G. Este fue su inicio para avanzar progresivamente hacia la tecnología digital 2G, $3 G$ y 4G, sufriendo cambios fundamentales, mejorando los servicios de conectividad y prestaciones de servicios. Las generaciones mencionadas presentaban diferencias en velocidades y características que mejora en la generación actual.

El avance de esta última generación 4G y con el IOT da paso a una generación mejorada llamada 5G, que permitirá nuevas experiencias de conectividad. Las redes de telecomunicaciones 5G llegarán con más velocidad, menos latencia, bajo consumo de energía y más prestaciones e interacciones con el IoT, satisfaciendo las necesidades de comunicación. Es claro que el despliegue comercial de la red 5G requerirá de la planificación, visibilidad y lanzamiento del espectro a largo plazo.
Es una necesidad la transformación de la red, la cual lleva bastante tiempo. 5G logrará su apertura para acelerar la innovación y el crecimiento tecnológico, es el catalizador para aumentar la productividad y eficiencia. El cambio a esta nueva comunicación $5 G$ y en conjunto con el IOT permitirá a futuro construir ciudades inteligentes y todo dispositivo estará conectado de forma eficiente. Así, se logrará una reducción de costos, ahorro de recursos naturales, optimización de procesos y recursos, sistematización de la industria, generación de negocios, entre otros.

El Proyecto Asociación de Tercera Generación (3GPP, 3rd Generation Partnership Project:), organización internacional que rige los estándares de comunicación móvil, aprobó la especificación técnica del estándar NSA 5G NR. Esta se apoyará en las redes existentes de 4G LTE lo que significa que la red $5 \mathrm{G}$ será compatible con la infraestructura $4 G$, ofreciendo portadoras de $5 G$ en la interfaz de aire. Lo anterior quiere decir que se debe mejorar la infraestructura existente para esta nueva red.

Asimismo se definió que para las zonas donde aún no hay infraestructura alguna, se implementará desde ceros el estándar SA, solo compatible con 5G, por esto es claro la transición de 5G NSA a 5G SA. Aún no es oficial pero se estima que el espectro a utilizar esta nueva tecnología estará en las bandas bajas con frecuencias estimadas entre 600 y 700 Mhz, en la banda media con frecuencias de uso de los 3.5Ghz y en la banda alta con frecuencias de $50 \mathrm{Ghz}$

Cabe aclarar que cada banda tendrá su propio cometido para garantizar el correcto funcionamiento de esta nueva red, una red implementada en la norma IEEE 802.11ac Wave 2. Esto logrará reducir las interferencias inalámbricas y mejorar la calidad de la señal. Hay muchas expectativas de esta nueva red móvil que revolucionará la forma de conectarnos y comunicarnos.

La nueva red móvil 5G revolucionará las comunicaciones inalámbricas trayendo consigo un aumento en el rendimiento de transferencia, velocidades de transferencia de datos en downlink de diez (10) Gbps, cobertura al 100\%, baja latencia de 1 a 4 milisegundo en la transmisión aproximadamente, consumo menor de energía, innovación y automatización de la industria y grandes avances en la conexión de dispositivos inteligentes con el loT. 
Para lograr un buen servicio de la red $5 \mathrm{G}$ se requiere de una gran inversión en infraestructura, ya sea inicial o mejorando la existente. Es decir, se debe mejorar la combinación de software y hadware, fortaleciendo ambos ámbitos para mejorar la cobertura y capacidad de conexión y soportar la nueva demanda. Para lograr las prestaciones de calidad de la 5G, es necesario contar con un gran espectro y ancho de banda con frecuencias de transmisión superiores a las actuales.

\section{LISTA DE REFERENCIAS}

[1] E. M. Ortega, "La telefonía móvil de cuarta generación 4G y Long Term Evolution", J. Ingenius, vol. 4, pp. 3-12, 2010.

[2] J.G. Andrews, et al., "¿Qué será 5G?", J. IEEE en áreas seleccionadas en comunicaciones, vol. 32, no. 6, pp. 1065-1082, 2014.

[3] M. Choudhary, "Tecnología 5G", In Conf. 2018 IEEE INTERCON.

[4] W. Sánchez, "5G NR- Features", In Conf. 2018 IEEE INTERCON.

[5] G. L. Méndez, P.E. Jojoa y V.A. Terré, "Sistemas MIMO con un elevado número de antenas: clave para la 5G inalámbrica", Revista Entramado, vol. 11, no. 2, pp. 250-261, 2015.

[6] E. M. Oproiu, I. Gimiga y I. Marghescu, "5G Fixed Wireless Access-Mobile Operator Perspective", In Conf. 2018 IEEE Int. Conf. Commun (COMM), pp. 357-360.

[7] "Plan Nacional 5G 2018-2020", Min. Energía, Turismo y Agenda Digital, España, 2017 [En línea]. Disponible en: http://www.mincotur.gob.es/telecomunicaciones/5G/Documents/plan_nacional_5g.pdf.

[8] A. Durán Barrado, "Estudio y caracterización del canal y de la propagación en ondas milimétricas, orientados a su utilización en redes de comunicaciones móviles 5G", tesis de maestría, Univ. Politécnica de Madrid, Madrid, España, 2017.

[9] E. Perahia y M.X. Gong, "LAN inalámbricas Gigabit: una descripción general de IEEE 802.11 ac y $802.11 \mathrm{ad}^{\prime \prime}$, J. ACM SIGMOBILE Mobile Computing and Communications Review, vol. 15, no. 3, pp. 23-33, 2011.

[10] R. Van Nee, "Rompiendo la barrera de gigabit por segundo con 802.11 ac", Rev. Comunicaciones inalámbricas IEEE, vol. 18, no. 2, 2011.
[11] J. Sánchez y M. Cuellar. Análisis de series de tiempo con métodos econométricos para el control de congestión en redes de comunicaciones. Bogotá: Editorial Fundación Universitaria San Mateo, 2018. Recuperado de https://palma.sanmateo. edu.co/ 